\title{
ALGEBRAIC DEFORMATIONS AND TRIPLE COHOMOLOGY
}

\author{
THOMAS F. FOX ${ }^{1}$
}

\begin{abstract}
The fundamental theorems of algebraic deformation theory are shown to hold in the context of enriched triple cohomology. This unifies and generalizes the classical theory.
\end{abstract}

The fundamental results in algebraic deformation theory connect the low order cohomology groups of an algebra $A$ with the existence of deformations of the algebra structure on $A$. The theory for associative algebras was initiated by Gerstenhaber in [6], where he also outlined techniques applicable to other special cases (see [7]). Here we give a unified treatment of the deformation theories for a broad class of algebra types using the enrichment over the category of coalgebras and triple cohomology (see [1] and [2]).

Let $R$ be a commutative ring and let $(T, \mu, \eta)$ be a triple on the category Mod-R. Recall that a $T$-algebra structure on an $R$-module $A$ is given by a map $\alpha: A T \rightarrow A$ satisfying the equations:

$$
(\alpha) T \cdot \alpha=\mu \cdot \alpha: A T^{2} \rightarrow A, \quad \eta \cdot \alpha=\mathrm{id}: A \rightarrow A .
$$

Following Gerstenhaber, we would like to investigate when a formal power series $\alpha+\Sigma \alpha_{n} x^{n}$ with coefficients in $\operatorname{Hom}_{R}(A T, A)$ determines a formal $T$-algebra structure on $A$ (or a $T$-algebra structure on $A[[x]]$, see [6]). Direct use of the conditions (1) immediately brings up the problem of the nonadditivity of most triples of interest, e.g. the tensor-algebra triple. To circumvent this problem we must use an additive enrichment of $T$ over the category of $R$-coalgebras. We shall assume that the algebras for $T$ are definable by a set of finitary multilinear operations (see [1] or [5]) which is the case for most categories of interest, e.g. associative algebras, commutative algebras, Lie algebras, etc.

Coalgebras and enrichments. Recall that an $R$-coalgebra $(C, \delta, \varepsilon)$ is an $R$-module $C$ equipped with maps $\delta: C \rightarrow C \otimes C$ and $\varepsilon: C \rightarrow R$ satisfying the equations $\delta \cdot(\mathrm{id} \otimes \delta) \simeq \delta \cdot(\delta \otimes \mathrm{id}), \delta \cdot(\mathrm{id} \otimes \varepsilon) \simeq \mathrm{id} \simeq \delta \cdot(\varepsilon \otimes \mathrm{id})$, and $\delta \cdot \tau=\delta$ where $\tau$ is the "twist" isomorphism $C \otimes C \rightarrow C \otimes C$, and the other isomorphisms are the obvious canonical ones. The category of all $R$-coalgebras and their structure preserving maps will be denoted Coalg. This is cartesian closed and serves as a base category over which most categories of $R$-algebras are enriched (see [1] or [5]).

\footnotetext{
Received by the editors June 1, 1977 and, in revised form, October 4, 1978.

AMS (MOS) subject classifications (1970). Primary 16A58, 18C15, $18 \mathrm{D} 15$.

${ }^{1}$ The author wishes to thank the Forschungsinstitut für Mathematik of the ETH for their hospitality, and Jon Beck and Michael Barr for suggesting that this work could be done.
} 
Note that the "cofree-coalgebra" functor is the right adjoint to the obvious forgetful functor from Coalg to Mod-R.

A point in a coalgebra $C$ is an element $f$ satisfying $f \delta=f \otimes f$ and $f \varepsilon=1$ in $R$. An $N$-deformation of a point $f_{0}$ is a sequence of elements $\left(f_{n}\right), 0<n<N$, in $C$ satisfying the equations:

$$
\left.f_{n} \delta=\sum_{i+j=n} f_{i} \otimes f_{j}, \quad f_{n} \varepsilon=0 \quad \text { (if } n>0\right) .
$$

An $\infty$-deformation of $f_{0}$ determines a family of formal points in $C$; for any $r$ in $R$, the sum $F=\sum_{0}^{\infty} f_{n} r^{n}$ satisfies $F \delta=F \otimes F$ and $F \varepsilon=1$. Given some sense of convergence in $C$, as in the analytic theory, these may determine actual points in $C$ (see [6]).

If $A$ and $B$ are $R$-modules, we denote by $(A, B)$ the cofree-coalgebra over $\operatorname{Hom}_{R}(A, B)$. The adjunction ${ }^{\wedge}:(A, B) \rightarrow \operatorname{Hom}_{R}(A, B)$ induces an evaluation map $A \otimes(A, B) \rightarrow B$, and we may view $(-,-)$ as a generalized Hom functor defining an enrichment of Mod-R over Coalg. Given a map $f: A \rightarrow B$, we say an element $g$ of $(A, B)$ represents $f$ if $\hat{g}=f$. There is unique point representing each map $A \rightarrow B$, but also for each $d: A \rightarrow B$ and each point $f$ in $(A, B)$ there is a 1-deformation of $f$ representing $d$.

If $A_{\alpha}$ and $B_{\beta}$ are $T$-algebras (i.e. $\alpha: A T \rightarrow A$ and $\beta: B T \rightarrow B$ ), there is a subcoalgebra of $(A, B)$ whose points precisely represent the $T$-algebra maps from $A_{\alpha}$ to $B_{\beta}$ (see [4]). We denote this coalgebra by $\left(A_{\alpha}, B_{\beta}\right)$; this is the Coalg-valued Hom from T-alg. If $d$ is a 1-deformation of $f$ in $\left(A_{\alpha}, B_{\beta}\right), \hat{d}$ is a derivation from $A_{\alpha}$ to $B_{\beta}$ along the algebra map $\hat{f}$.

Though generally there is no linear map $\operatorname{Hom}_{R}(A, B) \rightarrow \operatorname{Hom}_{R}(A T, B T)$ defining $T$, there is always a natural enrichment of $T$ over Coalg, $\mathcal{T}:(A, B) \rightarrow(A T$, $B T)$. If $f$ is a point in $(A, B)$, then $\hat{f} T=\widehat{f} \widehat{J}$. If $d$ is a 1-deformation of $f, d \mathscr{T}$ represents the unique $f T$-derivation from $A T$ to $B T$ (viewed as algebras) that coincides with $d$ on $A$.

If $A, B$, and $C$ are $R$-modules, the composition map in Coalg $\circ:(A, B) \otimes$ $(B, C) \rightarrow(A, C)$ induces a composition of deformations defined by convolution, i.e. if $f=\left(f_{n}\right)$ is a deformation in $(A, B)$ and $g=\left(g_{n}\right)$ is a deformation in $(B, C)$, we define $(f \cdot g)_{n}$ to be $\sum_{i+j=n} f_{i} \circ g_{j}$ in $(A, C)$.

The deformations. We define the coalgebra $S$ to be the equalizer of the following pair of maps in Coalg:

$$
(A T, A) \underset{\mu \cdot()}{\stackrel{() \mathscr{G}()}{\rightrightarrows}}\left(A T^{2}, A\right)
$$

where () $\mathscr{T} \cdot()=\delta \cdot(\mathcal{T} \otimes$ id $) \cdot \circ$. The points in $S$ precisely represent the $T$-algebra structures on $A$, since $T$ and $\mathcal{T}$ coincide on points. Thus an $\infty$-deformation $\left(\alpha_{n}\right)$ of $\alpha$ in $S$ is a deformation of $\alpha$ towards another $T$-algebra structure on $A$; the series $\alpha+\sum \hat{\alpha}_{n} x^{n}$ is a deformation of $\alpha$ in the sense of Gerstenhaber, as outlined at the beginning of this paper.

Let $\alpha$ be a point in $S$. Two $\infty$-deformations $\alpha_{*}=\left(\alpha_{n}\right)$ and $\alpha_{*}^{\prime}=\left(\alpha_{n}^{\prime}\right)$ of $\alpha$ in $S$ are said to be equivalent if there exists an $\infty$-deformation $a_{*}$ of the identity point in 
$(A, A)$ such that $\alpha_{*} \cdot a_{*}=a_{*} \mathcal{J} \cdot \alpha_{*}^{\prime}$. In this case, $a_{*}$ is a $T$-algebra isomorphism of the formal $T$-algebra structures defined by $\alpha_{*}$ and $\alpha_{*}^{\prime}$ on $A$ (the $\infty$-deformations of the identity forming a group under convolution). A deformation is trivial if it is equivalent to the deformation $(\alpha, 0,0, \ldots)$.

Now consider an $\infty$-deformation $\left(\alpha_{n}\right)$ in $S$. From (3) we have $\mu \cdot \alpha_{n}=\sum_{i+j-n} \alpha_{i}$ J $\cdot \alpha_{j}$ for each $\alpha_{n}$, i.e.

$$
\alpha_{n} \mathcal{T} \cdot \alpha-\mu \cdot \alpha_{n}+\alpha \mathfrak{T} \cdot \alpha_{n}=\sum_{\substack{i+j=n \\ i \neq 0 \neq j}} \alpha_{i} \mathfrak{T} \cdot \alpha_{j}
$$

The left side of the above is a coboundary, but to understand in what sense it is one, we must consider

The enriched cohomology complex. If $G$ is the cotriple on T-Alg associated with $T$, the $T$-algebra $A_{\alpha}$ generates a resolution of $T$-algebras $A_{\alpha} G^{*} \rightarrow A_{\alpha}$ (see [2]). Hom-ing into a $T$-algebra $B_{\beta}$ yields a complex of coalgebras $\left(A_{\alpha}, B_{\beta}\right) \rightarrow\left(A_{\alpha} G^{*}, B_{\beta}\right)$ which we may resolve in Mod-R. This defines the enriched cohomology groups of $A_{\alpha}$ with coefficients in $B_{\beta}$. Given a $T$-algebra map $\lambda: A_{\alpha} \rightarrow B_{\beta}$ and looking at the cohomology of the complex restricted to 1-deformations over $\lambda$ yields the usual triple cohomology groups, as defined by Barr and Beck in [2] (since $\lambda$ gives $B_{\beta}$ the structure of an $A_{\alpha}$-module and the 1-deformations correspond to $R$-linear derivations).

The complex $\left(A_{\alpha} G^{*}, B_{\beta}\right)$ is isomorphic (through adjointness, see [3]) to a "nonhomogeneous" complex of coalgebras, yielding the sequence of $R$-modules and boundary maps as follows:

$$
\begin{gathered}
(A, B) \stackrel{\partial^{0}}{\rightarrow}(A T, B) \stackrel{\partial^{1}}{\rightarrow}\left(A T^{2}, B\right) \stackrel{\partial^{2}}{\rightarrow}\left(A T^{3}, B\right) \ldots, \\
() \partial^{n}=\alpha \mathscr{T}^{n} \cdot()-\sum_{i=0}^{n-1}(-1)^{i} \mathfrak{T} \mu \mathscr{T}^{n-1-i} \cdot()-(-1)^{n}() \mathscr{T} \cdot \beta .
\end{gathered}
$$

Again, restricting to 1-deformations over $\lambda U=\xi \in(A, B)$ yields the Barr-Beck (B-B) cohomology groups of $A_{\alpha}$ with coefficients in $B_{\beta}$, here denoted $H_{\xi}^{n}(\alpha, \beta)$. Note that in the following paragraphs the words "cochain", "cocycle", and "coboundary" refer to the Barr-Beck concepts unless otherwise stated. If $\alpha=\beta$ and $\xi=$ id, then the cohomology groups are denoted $H^{n}(\alpha, \alpha)$.

The connection. Reexamining (2) and (4), we see that the first nonzero term in the deformation $\alpha_{*}=\left(\alpha_{n}\right)$ is a 1-cocycle, an element of $Z^{1}(\alpha, \alpha)$.

1. Proposition. Every $\infty$-deformation is equivalent to a deformation whose first nonzero term is not a coboundary.

Proof. Let $\alpha_{k}$ be the term in question. If $\alpha_{k}$ is a coboundary, let $x$ be the 0 -cochain such that $x \partial^{0}=\alpha_{k}$. Define an $\infty$-deformation $a_{*}=\left(a_{n}\right)$ of the identity by: $a_{k}=x$ and $a_{n}=0$ for $n \neq k$. Letting $\alpha_{*}^{\prime}=a_{*} \mathcal{T} \cdot \alpha_{*} \cdot a_{*}^{-1}$, we find that $\alpha_{n}^{\prime}=0$ for $n<k$, and $\alpha_{k}^{\prime}=\sum_{h+i+j-k} a_{h} \mathcal{T} \cdot \alpha_{i} \cdot a_{j}^{-1}=\alpha_{k}-x \partial^{0}=0 . \alpha_{*}^{\prime}$ is equivalent to $\alpha_{*}$ by construction; the result follows by induction.

2. Corollary. If $H^{1}(\alpha, \alpha)=0$ every deformation of $\alpha$ is trivial. 
We now ask when a 1-deformation may be extended to an $\infty$-deformation. More generally, suppose $\left(\alpha_{i}\right)_{i<n}$ is an $(n-1)$-deformation of $\alpha$. If $\alpha_{n}$ (extending the sequence in $S$ ) is to exist it must satisfy condition (4), i.e.

$$
\alpha_{n} \partial^{1}=-\sum_{\substack{i+j=n \\ n \neq 0 \neq j}} \alpha_{i} \mathcal{T} \cdot \alpha_{j}
$$

This sum is the obstruction in $\left(A T^{2}, A\right)$ to extending $\left(\alpha_{i}\right)_{i<n}$.

3. LEMMA. The obstruction to extending a truncated deformation is a cocycle in the enriched cohomology.

Proof. A trivial computation of $\partial^{2}$ acting on the obstruction, using (4) and the naturality of $\mu$.

Let obs $\left(\alpha_{n}\right)$ denote the obstruction defined above, and let $x_{n}$ be any element of $(A T, A)$ such that

$$
x_{n} \delta=x_{n} \otimes \alpha_{0}+\alpha_{0} \otimes x_{n}+\sum_{\substack{i+j=n \\ i \neq 0 \neq j}} \alpha_{i} \otimes \alpha_{j}
$$

(there are many of these because $(A T, A)$ is cofree). Then $x_{n} \partial^{1}-\operatorname{obs}\left(\alpha_{n}\right)$ is a 2-cochain, and in fact is a cocycle. The class of $x_{n} \partial^{1}-\operatorname{obs}\left(\alpha_{n}\right)$ (which does not depend on the choice of $x_{n}$ ) is the B-B obstruction. If $y$ is a 1-cochain such that $y \partial^{1}=x_{n} \partial^{1}-\operatorname{obs}\left(\alpha_{n}\right)$, then defining $\alpha_{n}=x_{n}+y$ extends $\left(\alpha_{i}\right)_{i<n}$, so we have

4. Proposition. A truncated deformation may be extended if and only if its $B-B$ obstruction vanishes.

5. Corollary. If $H^{2}(\alpha, \alpha)=0$ then every 1-cocycle extends to an $\infty$-deformation of $\alpha$.

Cohomology of the deformed algebra. Consider deformations $\alpha_{*}$ of $\alpha$ and $\beta_{*}$ of $\beta$, and a formal map $\xi_{*}=\left(\xi_{n}\right)$ from $\alpha_{*}$ to $\beta_{*}$, i.e. $\xi_{*}$ is an $\infty$-deformation of $\xi_{0}$ in $(A, B)$ and $\alpha_{*} \cdot \xi_{*}=\xi_{*} \mathcal{T} \cdot \beta_{*}$. Noting that $\alpha \cdot \xi_{0}=\xi_{0} \mathcal{T} \cdot \beta$ we may now compare $H_{\xi}^{n}\left(\alpha_{*}, \beta_{*}\right)$ with $H_{\xi}^{n}(\alpha, \beta)$; the classical results here (Gerstenhaber [8], Coffee [4]) state that $\operatorname{dim} H^{n}\left(\alpha_{*}, \alpha_{*}\right)<\operatorname{dim} H^{n}(\alpha, \alpha)$, where these are vector spaces over appropriate fields.

Now $H_{\xi_{*}}^{n}\left(\alpha_{*}, \beta_{*}\right)$ is defined by a complex such as (6) where $\alpha$ is replaced by $\alpha_{*}$, $\beta$ by $\beta_{*}$, and composition involves convolution of sequences. The boundary operator here will be denoted $\partial_{*}$, the cocycles $C_{*}$, etc.

Definition. An $N$-cochain (over $\xi_{*}$ ) is a sequence $\left(\xi_{1 n}\right)=\xi_{1 *}$ of elements in $\left(A T^{N}, B\right)$ such that

$$
\xi_{1 n} \delta=\sum_{i+j=n} \xi_{1 i} \otimes \xi_{j}^{N}+\xi_{j}^{N} \otimes \xi_{1 i}, \quad \xi_{1 n} \varepsilon=0 .
$$

(Here $\xi_{*}^{N}$ equals $\xi_{*}$ in dimension $0, \alpha_{*} \cdot \xi_{*}$ in dimension 1 , etc. We usually omit this superscript $N$.) $\xi_{1 *}$ is an $N$-cocycle if $\xi_{1 *} \partial_{*}^{N}=0$, i.e.

$$
\sum_{i+j=n} \alpha_{i} \mathcal{T}^{N} \cdot \xi_{1 j}-(-1)^{N} \xi_{1 j} \mathcal{T} \cdot \beta_{i}-\sum_{i=0}^{N-1}(-1)^{i} \mathfrak{T}_{\mu} \mathscr{T}^{n-1-i} \cdot\left(\xi_{1 n}\right)=0
$$


If $\xi_{1 *} \in Z_{*}$, in particular we have $\xi_{10} \delta=\xi_{10} \otimes \xi_{0}+\xi_{0} \otimes \xi_{10}$ and $\xi_{10} \partial=0$, i.e. $\xi_{10}$ is a cocycle over $\xi_{0}$. Thus $\xi_{1 *} \mapsto \xi_{10}$ defines a map $Z_{*} \rightarrow Z$, the image of which is the module of liftable cocycles, denoted $Z_{l}$. Obviously, this map carries coboundaries to coboundaries, so we have an induced map $H_{*} \rightarrow H$, the image of which is the module of liftable classes, denoted $H_{l}$.

Letting $Z_{n}=\left\{\xi_{1 *} \in Z_{*}: \xi_{1 i}=0, i<n\right\}$ defines a filtering $Z_{*}=Z_{0} \supset Z_{1} \supset$ $Z_{2} \ldots$ Notice that if $\xi_{1 *} \in Z_{*}$, then so is $\xi_{1 *}^{(k)}$ defined by

$$
\xi_{1 n}^{(k)}= \begin{cases}0, & n<k \\ \xi_{1(n-k)}, & n \geqslant k\end{cases}
$$

this defines an isomorphism $Z_{0} \stackrel{\sim}{\rightarrow} Z_{k}$. Now it is obvious that $Z_{l} \simeq Z_{0} / Z_{1}$, and in fact $Z_{l} \simeq Z_{n} / Z_{n+1}$ for every $n \geqslant 0$.

6. Proposition. Let $H_{*}=H_{0} \supset H_{1} \supset H_{2} \ldots$ be the induced filtering of $H_{*}$. Then there are epimorphisms $H_{l} \rightarrow H_{n} / H_{n+1}$ for all $n>0$.

Proof. Again we have $H_{l} \simeq H_{0} / H_{1}$, and the map $H_{0} \rightarrow H_{k}$ induced by $Z_{0} \rightarrow Z_{k}$ is an epimorphism.

Note that the epimorphisms mentioned above may not be monomorphisms. Pertinent to this is the question of when an $n$-truncated $N$-cocycle $\left(\xi_{1 i}\right)_{i<n}$ may be extended to an element of $Z_{*}^{N}$. If the continuation, $\xi_{1 n}$, is to exist it must satisfy (7) and (8), from which we get

$$
\sum_{\substack{i+j=n \\ i \neq 0}}-\alpha_{i} \mathscr{T}^{N} \cdot \xi_{1 j}+(-1)^{N} \xi_{1 j} \mathcal{T} \cdot \beta_{i}=\xi_{1 n} \partial^{N} .
$$

The sum on the left of the above equation is the obstruction, obs $\left(\xi_{1 n}\right)$, in $\left(A T^{N+1}\right.$, $B)$ to extending $\left(\xi_{1 i}\right)_{i<n}$. It is easy to show that the obstruction is a cocycle in the enriched cohomology. As in the case of a truncated deformation, it defines a class in $H^{N+1}$ which must vanish if $\xi_{1 n}$ is to exist. More precisely, let $x_{1 n}$ be any element of $\left(A T^{N}, B\right)$ such that

$$
x_{1 n} \delta=x_{1 n} \otimes \xi_{0}+\xi_{0} \otimes x_{1 n}+\sum_{\substack{i+j=n \\ i \neq 0}} \xi_{1 i} \otimes \xi_{j}+\xi_{j} \otimes \xi_{1 i} .
$$

Then the class of $x_{1 n} \partial-\operatorname{obs}\left(\xi_{1 n}\right)$ in $H^{N+1}$ is the B-B obstruction.

7. Proposition. If an n-truncated cocycle is obstructed, then the epimorphism $H_{l} \rightarrow H_{n} / H_{n+1}$ has a nontrivial kernel.

Proof. Consider any element $\lambda_{1 *} \in C_{*}$ such that

$$
\lambda_{1 i}= \begin{cases}\xi_{1 i}, & i<n, \\ x_{1 n}, & i=n,\end{cases}
$$

and look at $\lambda_{1 *} \partial_{*}$; it is easy to see that its first $n-1$ terms are 0 while its $n$th term is $x_{1 n} \partial-\operatorname{obs}\left(\xi_{1 n}\right)$. Thus $x_{1 n} \partial-\operatorname{obs}\left(\xi_{1 n}\right) \in H_{l}$ but its image in $H_{n} / H_{n+1}$ is the class of $\lambda_{1 *} \partial_{*}$. 


\section{REFERENCES}

1. M. Barr, Coalgebras over a commutative ring, J. Algebra 8 (1974), 600-610.

2. M. Barr and J. Beck, Homology and standard construction, Lecture Notes in Math., vol. 80, Springer-Verlag, Berlin and New York, 1969, pp. 245-334.

3. J. Beck, Triples, algebras and cohomology, Dissertation, Columbia University (1967).

4. J. P. Coffee, Filtered and associated graded rings, Bull. Amer. Math. Soc. 78 (1972), 584-587.

5. T. F. Fox, Universal coalgebras, Dissertation, McGill University (1977).

6. M. Gerstenhaber, On the deformation of rings and algebras. I, Ann. of Math. (2) 79 (1964), 59-103.

7.

8. On the deformation of rings and algebras. IV, Ann. of Math. (2) 99 (1974), 257-276.

Department of Mathematics, Dawson College, 1001 Sherbrooke East, Montreal, Qubbec, Canada 